\title{
Indicadores de calidad en colangiografía endoscópica y ultrasonido endoscópico
}

\author{
Ángela Saúl-Pérez* \\ Instituto Nacional de Ciencias Médicas y Nutrición Salvador Zubirán, Ciudad de México, México
}

\section{Resumen}

En 2015 la Sociedad Americana de Endoscopia Gastrointestinal (ASGE) y más recientemente en 2018 la Sociedad Europea de Endoscopia Gastrointestinal (ESGE), publicaron guías de calidad en colangiopancreatografía retrógrada endoscópica (CPRE) y Ultrasonido Endoscópico (USE). En dichos documentos se establecen lineamientos que cada centro debe mantener en base a porcentajes pre-establecidos. Este año en la Semana de Enfermedades Digestivas (DDW) se revisó el estado actual de dichos lineamientos así como sus perspectivas a futuro. A lo largo de este texto se sintetizarán los puntos más sobresalientes de los trabajos presentados.

Palabras clave: Colangiopancreatografía retrógrada endoscópica (CPRE). Ultrasonido endoscópico (USE). Estándar de calidad.

Dentro de las conferencias más relevantes respecto a este tema, destacó la impartida por Rajesh Keswani de Northwestern University quien recalcó la importancia de medir la calidad de la CPRE y USE y como el éxito de estos procedimientos depende de la habilidad, entrenamiento y desempeño del médico que realiza el estudio.

En 2015, la Sociedad Americana de Endoscopia Gastrointestinal (ASGE) $)^{1,2}$ y, más recientemente, en 2018, la Sociedad Europea de Endoscopia Gastrointestinal (ESGE) ${ }^{3}$ publicaron guías de calidad en CPRE y USE. En dichos documentos se establecen lineamientos que cada centro debe mantener en base a porcentajes pre-establecidos. Medir los estándares de calidad es el pilar fundamental para lograr mejorar el desempeño en estas áreas de la endoscopia avanzada, sin embargo es importante determinar si es factible y relevante medir dichos estándares de calidad. A este respecto y de acuerdo a Rajesh Keswani, en la CPRE el primer indicador de calidad es la indicación del procedimiento. Las guías de la ASGE recomiendan que más del $90 \%$ de las CPRE's deben tener una indicación documentada sin embargo en la práctica real esto es poco factible y relevante. Por otro lado la tasa de canulación sin anatomía modificada recomendada por las guías es superior al 90\% considerando a esta medida de calidad factible de medir y relevante. Otro estándar de calidad es la extracción de litos biliares menores a $1 \mathrm{~cm}$ de diámetro en un conducto biliar normal y así como la colocación de prótesis en estenosis distales por debajo de la confluencia de los hepáticos, ambas recomendadas en $\geq 90 \%$ de los casos. Este control de calidad es relevante ya que $2 / 3$ partes de las CPRE's realizadas son debidas a estenosis o litiasis, sin embargo no es fácil de determinar con certeza. En relación a la pancreatitis post-CPRE la medición de

\section{Correspondencia:}


esta como indicador de calidad es factible y relevante sin embargo no hay un porcentaje "blanco" ya que la tasa de esta complicación varía de acuerdo a la indicación del procedimiento.

Wenkang $\mathrm{Fu}$, et al. presentaron una revisión sistemática y meta-análisis del número apropiado de CPRE's que debe realizar un residente en formación para obtener una competencia básica. La ASGE establece que la tasa de canulación debe ser $>90 \%$ considerándola como indicador de calidad, sin embargo en este estudio de 149 residentes y 18,794 CPRE's, la tasa de canulación lograda fue de $85.7 \%$ luego de haber realizado 200 procedimientos, aumentando muy poco luego de más de 200 procedimientos.

Respecto al USE, los estándares de calidad tales como el uso de sistema de TNM para etapificar neoplasias, así como la sensibilidad y rendimiento diagnóstico de la biopsia de lesiones pancreáticas (las guías recomiendan sea $>85 \%$ ) ambas son factibles y relevantes. Mientras que los eventos adversos de una biopsia guiada por USE es probablemente factible y de poca relevancia debido a la baja incidencia de esta complicación. Lawrence Ku y colaboradores de UCLA reportaron su experiencia en la evaluación retrospectiva de los indicadores de calidad en USE en un periodo de un año donde destaca un apego a las guías de ASGE de $99.5 \%$ en relación a la indicación del procedimiento. Interesantemente ellos describen el impacto del USE medido como un cambio en el manejo postUSE en el $59 \%$ de los casos ( $31 \%$ para coledocolitiasis y $80 \%$ para malignidad). La tasa de complicaciones descrita fue de $0.68 \%$.

La mayoría de los endoscopistas no mide estos estándares de calidad en su práctica cotidiana ya que consumen mucho tiempo por lo que una de las recomendaciones a futuro es mejorar el sistema de medición, ajustando el riesgo por paciente y por indicación así como determinar los desenlaces de éxito y complicaciones a corto y largo plazo e incluso llevar a cabo una valoración video asistida de las destrezas técnicas por otro colegas de las misma especialidad. Referente a este tema, un grupo de expertos entre los que destacan Todd Baron y Shyam Varadarajulu entre otros presentaron un instrumento de validación video asistida para valorar las destrezas técnicas en CPRE donde evaluaban puntos como la estabilidad del duodenoscopio, la posición de la papila, la trayectoria de la guía, el control y dirección de la esfinterotomía entre otros además de determinar un "nivel" al desempeño del endoscopista.

Finalmente y concerniente a la sedación, las guías ASGE recomiendan contar con un plan de sedación establecido en $>98 \%$ de los casos. En la DDW se presentó un trabajo realizado por Marco Albuquerque, et al., de España donde compararon la calidad de la sedación en CPRE en relación a quien administró la sedación (anestesiólogos, intensivistas y endoscopistas). En base a esto determinaron la tasa de efectos adversos, el número de procedimientos cancelados y la calidad de la imagen radiológica determinada por la posición del paciente. Evaluaron 454 pacientes y encontraron que la tasa de eventos adversos fue menor para aquellos pacientes sedados por endoscopistas mientras que la tasa de procedimientos cancelados y peor calidad de imagen fluoroscópica se presentó en el grupo sedado por anestesiólogos. La cantidad de pacientes con baja saturación de $\mathrm{O} 2$ y en quienes se requirió de alguna maniobra de resucitación respiratoria fue mayor en el grupo de anestesiólogos e intensivistas.

A manera de conclusión en la DDW 2019 se recalcó la importancia de apegarse a los estándares de calidad en CPRE y USE, así como también se inició la investigación hacia sistemas automatizados para reportar y valorar dichos estándares y sistemas de validación que perfeccionan dicha valoración.

\section{Conflicto de intereses}

No existen conflictos de interés. Sin patrocinio de la industria.

\section{Bibliografía}

1. ASGE Guidelines. Quality indicators for ERCP. Gastrointest Endosc 2015; 81: 54-66.

2. ASGE Guidelines. Quality indicators for EUS. Gastrointest Endosc 2015; 81: 67-80.

3. Domagk D, Oppong KW, Aabakken L et al. Performance measures for endoscopic retrograde cholangiopancreatography and endoscopic ultrasound: A European Society of Gastrointestinal Endoscop (ESGE) Quality Improvement Initiative. United European Gastroenterol J. 2018; 6 (10): 1448-1460. 